\title{
La Pensée matérialiste et la conception sensualiste de Denis Diderot dans Lettre sur les aveugles à l'usage de ceux qui voient
}

\begin{abstract}
par
Dr. Siham Abd El Fattah Mohamed Gabr*

Diderot a reçu une instruction solide dès l'âge de 18 ans. Cette instruction sera à la base de sa prodigieuse culture. Dans sa Lettre sur les aveugles à l'usage de ceux qui voient, Diderot essaye de lancer une conception de notre monde, une nouvelle méthode ou pour mieux dire une théorie d'ensemble ; il pense arriver à des résultats fragmentions apportés par les nouvelles sciences de la nature. Diderot, comme nous l'avons remarqué ,conteste dans son œuvre, les preuves traditionnelles de l'existence de Dieu ; car que valent pour un aveugle l'ordre du monde et les merveilles de la nature.
\end{abstract}

Malgré tout, nous pouvons dire que Diderot ne compte pas de philosophies le matérialisme ou le sensualisme quoiqu'il lance une théorie ou une conception basée sur le matérialisme et le sensualisme; plus fondée et plus sure que les idées des penseurs déjà cités .

\footnotetext{
* Professeur adjoint de la littérature française -Faculté des lettres Université de ZAGAZIG
}

(La Pensée matérialiste et la conception sensualiste) Dr. Siham Gabr 
C'est, en effet ; il va de soi que les idées de Diderot exprimées dans cette lettre ont été contestées et discutées par plusieurs autres penseurs. De telles critiques exigeront, bien sûr, d'autres recherches d'une plus grande envergure.

\section{Introduction}

Diderot a reçu une instruction solide dès l'âge de 18 ans. Cette instruction sera à la base de sa culture prodigieuse. En effet, Diderot a été impérieusement attiré vers la culture désintéressée et s'est initié aux formes les plus variées de la pensée humaine. Sa relation pour plusieurs années avec l'aventurière Mme de Puisieux a joué un certain rôle important dans sa production littéraire et philosophique surtout. Il devient un polygraphe dans la période qui s'étend de 1746 à 1773. Il produit un nombre considérable d'ouvrages de tous genres qui étaient souvent peu connus ou même inconnus de ses contemporains et dont certains n'étaient pas publiés.

Cependant, il faut dire que cette activité culturelle a été, un moment, contrariée en 1749 après la publication de la Lettre sur les aveugles à l'usage de ceux qui voient ; car la hardiesse de sa pensée qui, déjà s'inclinait à l'athéisme et au matérialisme, lui a valu d'être emprisonné pendant trois mois au Donjon de Vincennes.

Dans l'ouvrage que nous étudions : Lettre sur les aveugles à l'usage de ceux qui voient, Diderot s'adresse à Mme de Puisieux, son amie pendant plusieurs années et qui adorait la littérature et la philosophie. Diderot prend, dans cet

(La Pensée matérialiste et la conception sensualiste) Dr. Siham Gabr 
ouvrage, comme point de départ, l'opération de la cataracte que Réamur ${ }^{(1)}$ venait de pratiquer et de réussir sur un prétendu aveugle né. Diderot s'élève à des considérations générales sur la cécité. Puis il prête au mathématicien Anglais Saunderson, qui avait perdu sa vue à l'âge d'un an, ses argumentations en faveur de l'athéisme ; toute connaissance vient des sens. Pour lui alors, l'ordre mondiale ne saurait être une preuve de l'existence de Dieu, et cet ordre est, d'ailleurs, loin d'être parfait puisqu'il admet des monstres inadaptés à la vie. Il est particulièrement difficile à un aveugle de croire en la divinité. D'autre part, un aveugle se passe de cette croyance sans importance pour lui.

Nous allons donc traiter dans notre recherche, deux points importants : la pensée matérialiste et la conception sensualiste pour essayer de cristalliser la croyance de cet écrivain.

\section{I_-La pensée matérialiste .}

Dans sa Lettre sur les aveugles à l'usage de ceux qui voient, Diderot essaye de lancer une conception de notre monde, une nouvelle méthode ou pour mieux dire une théorie d'ensemble ; il pense arriver à des résultats de fragmentions apportés par les nouvelles sciences de la nature. Il a tiré une idée très simple quoique géniale qui n'est autre que la présupposition la plus générale de ces sciences : à savoir

(1) -René-Antoine Ferchault de Réaumur, né le 28 février 1683 à La Rochelle et mort le 17 octobre 1757 dans son domaine de la Bermondière, à SaintJulien-du-Terroux, en Mayenne, est un physicien et naturaliste français. Wikipédia

(La Pensée matérialiste et la conception sensualiste) Dr. Siham Gabr 
l'existence de la nature matérielle, de son devenir et de ses lois.

La notion qui permet à Diderot de dépasser à la fois, le mécanisme brut et la métaphysique finaliste, se trouve dans la notion du devenir dans la nature. Cette notion inclut une autre, celle de la sélection des formes des espèces ou des êtres dans la nature. Comment s'expliquent l'ordre, l'harmonie, et l'apparente finalité des êtres que tout le monde constate? .En effet, Diderot considère toutes les combinaisons possibles des parcelles ou molécules des matières. D'après lui, les combinaisons harmonieuses s'accomplissent dans la nature et se succèdent comme dans un jeu humain, c'est-à-dire selon lui les combinaisons heureuses, bien qu'ayant peu de chance de se réaliser, dès qu'elles sont réalisées, elles ont aussitôt beaucoup de chances de durer. L'esprit humain constate alors que telle combinaison est heureuse au fait qu'elle dure. Selon Diderot la nature est un chaos de forces et de mouvements ; c'est un ensemble de contradictions et de conflits. À ce propos, l'auteur dit :

"...que nous sentissions la matière se mouvoir et le chaos, se débrouiller, nous rencontrerions une multitude d'êtres informer pour quelques êtres bien organisés ${ }^{\prime(1)}$

La forme "heureuse" se maintient parmi ces conflits et elle y évolue et les formes de son développement ont aussitôt un nombre croissant de chances de réalisation.

(1) - Diderot(Denis): Lettre sur les aveugles ..., Textes établis par Paul Vernière. Paris.Garnier 1963., p. 121

(La Pensée matérialiste et la conception sensualiste) Dr. Siham Gabr 
Les autres restent seulement possibles parmi les possibles infinis :

"......que toutes les combinaisons vicieuses de la matière ont disparu, et qu'il n'est resté que celles ou le mécanisme n'impliquait aucune contradiction importante, et qui pouvaient subsister par elles-mêmes et se perpétuer....." ${ }^{\prime(1)}$

Si le premier homme n'eût pas présenté une de ces formes heureuses, les hommes, ces êtres orgueilleux seraient restés dissous, dispersés entre les molécules de la matière et peut-être pour toujours au nombre des possibles :

"...que devenait le genre humain ? il eût été enveloppé dans la dépuration générale de l'univers ; et cet être orgueilleux qui s'appelle homme, dissous et dispersé entre les molécules de la matière, serait resté, peut-être pour toujours, au nombre des possibles." (2)

Dans cet entretien entre l'aveugle Saunderson et le prêtre Holmes sur l'existence de Dieu, lequel compte parmi l'une des pages les plus puissantes que Diderot n'ait jamais écrites Diderot ; il s'élève à une conception de la matière et de la nature toujours plus complexe, ou faisant intérieure la notion alors toute nouvelle, d'évolution, ainsi que celle du calcul des probabilités. Il atteint, dans le cadre des connaissances que lui offrait son époque aux confins de cet univers, s'adressant alors aux philosophes ; il les défie de

\footnotetext{
(1) - Diderot(Denis): Lettre sur les aveugles ..., Textes établis par Paul Vernière.

Paris.Garnier 1963... pp. 121-122

(2)

Ibid.: p.122
}

(La Pensée matérialiste et la conception sensualiste) Dr. Siham Gabr 
trouver, à travers les agitations irrégulières de ce nouvel Océan de matière s'ouvrant sous leurs pieds "quelques vestiges de cet être intelligent dont vous admirez ici la sagesse" ${ }^{(1)}$

À ce point extrême de sa pensée, Diderot nous apparaît comme un des chaînons essentiels qui, dans la lignée des philosophes matérialistes, relie le matérialisme scientifique moderne ou dialectique. Il va de soi que le matérialisme considère la matière comme la seule réalité et nie l'existence de l'âme, de l'au-delà et de Dieu.

C'est ainsi que Diderot, essaye dans sa Lettre sur les aveugles, de définir le monde par le biais de Saunderson : "qu'est-ce que ce monde, monsieur Holmes? Un composé sujet à des révolutions qui, toute, indiquent une tendance continuelle à la destruction ${ }^{\prime \prime(2)}$

D'après lui, le monde est l'espace où "des mondes se reforment et se disposent, peut-être, à chaque instant ... un monde où le mouvement continue et continuera à combiner des âmes de matières ${ }^{\prime \prime(3)}$ Mais ce mouvement créateur est un mouvement destructeur qui déferait "les âmes de matières qu'il a combinés...le bloc de marbre tend à sa dissolution..." ${ }^{\prime(4)}$

\footnotetext{
(1) - Diderot(Denis): Lettre sur les aveugles ... , Textes établis par Paul Vernière. Paris.Garnier 1963., p. 123

(2) - Idem.

(3) -Idem.

(4) _Diderot(Denis): (Euvres complètes. Paris , Garnier, 1877.p.394
}

(La Pensée matérialiste et la conception sensualiste) Dr. Siham Gabr 
À l'idée du développement des espèces. Les notions de développement et d'accroissement voisinent avec la notion de dépérissement et d'accroissement.

Diderot conçoit cet instant du cosmos où le mouvement aurait épuisé tout son potentiel d'énergie où la dégradation de l'énergie induirait le désordre de la matière pour aboutir à son immobilité totale. Diderot a esquissé, à l'avant-garde de la pensée scientifique, la notion d'estropie voisinant avec celle de l'évolution. Diderot étend l'évolution à la biosphère entière, aux animaux comme aux étoiles. L'évolution est partout présente. Elle est inhérente à la matière sous toutes ses formes. Elle est coefficient de la matière, résultant de son existence énergique dans le temps.

À ce propos, il dit :- "Mais pourquoi n'assurerai-je pas des mondes ce que je crois des animaux? Combien de mondes estropiés, manqués se sont dissipés, se reforment et se dissipés, peut -être à chaque instant dans des espaces éloignés. ${ }^{(1)}$

En effet, Diderot conçoit l'évolution de l'univers comme celle de tous les systèmes dont il est composé.

D'autre part, son idée de l'existence d'un invariant sous l'accumulation de la variété de ses manifestations, se retrouve souvent chez lui. Cette réflexion est claire dans sa Lettre sur

${ }^{(1)}$ Diderot(Denis): Lettre sur les aveugles ... , Textes établis par Paul Vernière. Op.cit, p. 123

(La Pensée matérialiste et la conception sensualiste) Dr. Siham Gabr 
les aveugles où il pense que "le temps, la matière et l'espace ne sont, peut-être qu'un point." (1)

L'équation temps-matière-espace suggère que le temps et l'espace sont les deux modes inverses d'existence de la matière. Disons encore que la prise de conscience, au XVIIIème siècle, de l'existence réelle du mouvement de la matière fait de Diderot un matérialiste : il va plus loin en donnant à Dieu une autre dimension ou une autre définition en l' identifiant avec le mouvement de la matière au lieu de le considérer comme agent du mouvement.

Diderot, partant de son principe fondamental, voit que la matière est divisée en matière morte et en matière vivante. Dans son autre ouvrage intitulé De l'interprétation de la nature, il écrit : -" S'il y a une matière morte, par ellesmêmes, ces deux principes suffisent -ils pour la production générale de toutes les formes et de tous les phénomènes?" (2)

En formulant cette question, il essaie de déterminer la nature de la contradiction, source suggérée du mouvement créateur et destructeur en même temps.

D'ailleurs, la distinction entre la matière vivante et la matière morte demeure le point de départ d'où émergeront les hypothèses donnant à la vision du monde de Diderot, une extraordinaire armature dont on n'a pas encore réussi à dénombrer toutes articulations. S'il lui est difficile de concevoir les rapports entre la matière vivante et la matière

\footnotetext{
(1) - Diderot(Denis): Lettre sur les aveugles ... , Textes établis par Paul Vernière. Op.cit, p. 123

(2) _Diderot(Denis): De l'interprétation de la nature, Paris . Garnier.1964 .p.242
}

(La Pensée matérialiste et la conception sensualiste) Dr. Siham Gabr 
morte, Diderot pense que la sensibilité devient commune à la masse commune puisque cette sensibilité établit un premier lien entre l'animé et l'inanimé. D'après l'écrivain, les bords de l'abîme entre la matière vivante et la matière morte "ne rapprochent puisqu'une substance dans l'univers dans l'homme, dans l'animal." (1)

Diderot pense que la distance entre le marbre et l'homme par exemple, se réduit ; car tous les deux sentent ; ce qui les différencie, c'est de sentir à des degrés différents et déterminés par l'organisation. C'est ainsi que la sensibilité est commune à la matière quoiqu'elle varie en intensité. C'est ainsi que l'intelligence considérée par Diderot comme une manifestation de la sensibilité à son haut degré de réalisation est unie à des portions de matière très énergétique. Donc, l'univers entier s'érige dur la molécule.

En effet, Diderot conçoit, en sous d'une matière sensible, une matière moléculaire qui pourrait éventuellement expliquer la différence d'organisation qui existe, par exemple entre le cristal et l'animal ; entre l'animé et l'inanimé.

D'autre part, nous devons signaler que l'évolutionnisme de Diderot comprend certainement l'idée du mécanisme, mais les transformations ne résultent guère d'une interaction entre le milieu et l'organisme.

On peut, d'après les idées exprimées dans la Lettre sur les aveugles à l'usage de ceux qui voient, dire que le matérialisme de cet écrivain, est difficile à comprendre au

(1) _Diderot(Denis): Entretien entre D'Alembert et Diderot , Paris . Garnier.1966 .p. 279

(La Pensée matérialiste et la conception sensualiste) Dr. Siham Gabr 
XVIIIème siècle, comprend trois hypothèses puisque le monde n'est que -d'après lui- matière et mouvement. Il y a de la matière morte et de la matière vivante.

Diderot suppose donc, et c'est la première hypothèse capitale de sa pensée exprimée dans cette lettre, que la matière est douée d'une sensibilité qui, dans certains cas, est latente et dans d'autres vivante : "que nous aurions de peine, sans nos yeux, à supposer qu'un bloc de marbre ne pense ni ne sent" ${ }^{(1)}$

On trouve que la deuxième hypothèse suppose que la matière s'organise d'elle-même et la finalité qui semble préside à l'existence du monde et des êtres peut très bien n'être que le résultat de combinaison en nombre infini de tentatives de la nature dont la plupart n'ont pas été viables et ont disparu à jamais. Sous le masque de Sounderson, Diderot a lancé cette hypothèse des monstres avortés : "... que les monstres se sont anéantis successivement, que toutes les combinaisons vicieuses de la matière ont disparu" (2)

Dans la troisième hypothèse, on découvre que Diderot est entrainé par cette évolution et ce flux de l'individu comme un maillon de la grande chaîne de l'espèce ; et même la matière, par un travail permanent, fond la création en un tout en perpétuel devenir : "Et cet être orgueilleux qui s'appelle homme, dissous et dispersé entre les molécules de la matière,

(1) -Diderot(Denis): De l'interprétation de la nature, op,cit. p. 144

(2) -Diderot(Denis): De l'interprétation de la nature , op,cit. p. 121

(La Pensée matérialiste et la conception sensualiste) Dr. Siham Gabr 
serait resté peut-être pour toujours, au nombre des possible. "(1)

C'est ainsi qu'on pourrait croire que Diderot a formulé, dans sa Lettre sur les aveugles à l'usage de ceux qui voient, les premières lois de la dialectique : unité de la nature donnée dans sa matérialité, caractère universel du devenir, de la naissance et de la disparition des choses et des mondes.

\section{II-La conception sensualiste:}

Nous savons, certes, que la sensualisme est une philosophie d'après la quelle toutes les idées proviennent des sensations.

C'est ainsi que Diderot a abandonné les entités abstraites et la forme abstraite des rapports.

La Lettre sur les aveugles à l'usage de ceux qui voient, affirme que la sensation ne réside pas dans les sens euxmêmes ; elle n'est pas non plus l'acte, abstraitement analysable appelé perception ; mais elle est liée concrètement au sens et aux objets.

Son sens du concret interdit à Diderot de poser, abstraitement le problème de la connaissance. Sa propre conception consiste à ramener vers le concret les faits et les attitudes pratiques. C'est la raison pour laquelle, il a laissé de côté les discussions interminables sur une question presque insoluble et d'intérêt secondaire : Les aveugles-nés à qui l'on rend la vue perçoivent-ils l'espace à trois dimensions? Ce

(1) -Ibid. p.. 122

(La Pensée matérialiste et la conception sensualiste) Dr. Siham Gabr 
qui intéresse Diderot, c'est le monde d'un aveugle adulte normal.

Un tel aveugle est un monstre, mais un monstre assez proche de nous pour nous apprendre beaucoup sur nousmêmes. Il réalise une sorte d'expérience naturelle : le monde de cet aveugle diffère assez du nôtre pour nous transmettre des renseignements, et comparable à celui de l'homme habituel. Diderot va à Puiseaux, petit village du Loiret, pour rendre visite à un aveugle qui ne manque ni le bon sens, ni l'éducation, ni même les connaissances. Mais, tout d'abord, que découvre Diderot?

Diderot ne manque pas cette expérience qu'il entoure d'hypothèses ou d'explications aussi ingénieuses que solides. Il constate tout d'abord que cet aveugle de Puiseaux possède, comme les autres, la faculté de juger. Il sait enchaîner ses discours, définir les mots et choisir se idées. Certes, le registre d'idées dont il dispose est moindre que celui dont jouent les hommes normaux.

Diderot remarque ensuite que, si les notions mathématiques et géométriques sont accessibles à cet aveugle de Puiseaux, "il ne lui combine que des points palpables." ${ }^{(1)}$

De cette observation, découle ce fait remarquable que, dans le domaine des idées et des abstractions, l'aveugle aperçoit les choses d'une manière plus abstraite que nous. Il semble donc qu'en matière de spéculation qu'il est moins sujet à se

(1) _Diderot(Denis): Lettre sur les aveugles ..., op.cit.P.95

(La Pensée matérialiste et la conception sensualiste) Dr. Siham Gabr 
tromper que l'homme clairvoyant: "L'abstraction ne consiste qu'à séparer par la pensée les qualités sensibles des corps, ou les unes des autres ou du corps même qui leur sert de base." (1) Diderot sait, en effet, qu'à son infirmité l'aveugle a la même organisation que les autres êtres humains : "Je n'ai jamais douté que nos idées les plus purement intellectuelles, si je puis parler ainsi, ne tiennent de fort près à la conformation de notre corps." (2) L'aveugle aura peut-être moins d'idées que le voyant ou des idées incomplètes et fausses sur certains points; il aura cependant des idées et saura les comparer et les combiner : "...Il est maître de combiner ces sensations ou points et d'en former des figures." ${ }^{\prime(3)}$

Diderot est convaincu que toutes les connaissances humaines partent des sens, comme cet aveugle qui traduit ou exprime tout en termes de contact, de toucher qui d'ailleurs possède la mémoire des sens à un degré surprenant et qui fait entrer dans ses jugements jusqu'aux sons et aux charmes des voix : "Il juge de la beauté par le toucher ........c'est qu'il fait entrer dans ce jugement la prononciation et le son de la voix $^{\prime \prime(4)}$

Mais les sens ne suffissent pas à définir la connaissance, car celle-ci exige des abstractions, des signes
(1) -Ibid. p.98
(2) -Ibid.p.92
(3) - Diderot(Denis): Lettre sur les aveugles ... _op.cit pp.94.95
(4) - Ibid. .p.90

(La Pensée matérialiste et la conception sensualiste) Dr. Siham Gabr 
et des termes qui ne peuvent être représentés par des objets sensibles :

"Nous ne parvenons à attacher une idée à quantité de termes qui ne peuvent être représentés par des objets sensibles et qui, pour ainsi dire, n'ont point de corps que par une suite de combinaisons fins et profondes des analogies que nous remarquons entre ces objets. ${ }^{(1)}$

Cette grande opération de la connaissance dépend donc de notre toucher, de notre vue et de toutes les sensations de notre corps : "Je conclus de là que nous tuons sans doute du concours de nos sens et de nos organes de grands services." (2) . Diderot pense que, dans les affaires de pure convention, l'aveugle juge bien comme le clairvoyant. Mais son monde esthétique diffère du nôtre : "La beauté, pour un aveugle n'est qu'un mot quand elle est séparée de l'utilité"(3)

C'est ainsi que l'aveugle de Puiseaux bien en employant ce mot et d'autres qui n'ont pour lui aucun sens ou qui ont un sens différent du nôtre. Il est semblable en cela à beaucoup d'hommes, et même à beaucoup de philosophes. Quant à son monde moral, il doit aussi différer du notre, de ce fait qu'il ne verra jamais couler le sang, ne sera affecté que par la plainte et manquera d'humanité : "Les aveugles ne sont affectés que par la plainte. Je les soupçonne en général, d'humanité. Quelle différence y a-t-il, pour un aveugle, entre

\footnotetext{
(1) - Ibid . p.91

(2) - Diderot(Denis): Lettre sur les aveugles ... ,op.cit.p.91

(3) - Ibid., p. 93
}

(La Pensée matérialiste et la conception sensualiste) Dr. Siham Gabr 
un homme qui urine et un homme qui, sans se plaindre, verse son sang "(1)

Pourtant de ses sensations, l'aveugle doit parvenir aux notions mathématiques et géométriques. Il rapporte tout à l'extrémité de ses doigts : " L'aveugle-né rapporte tout à l'extrémité de ses doigts." (2)

Que lui a montré donc l'aveugle de Puiseaux dont le monde esthétique et moral diffère du nôtre ? Diderot s'adresse à Mme de Puiseaux en disant :" Ah madame ! que la morale des aveugles est différente de la nôtre! que celle d'un sourd différerait encore de celle d'un aveugle, et qu'un être qui aurait un sens de plus trouverait notre morale imparfaite, pour ne rien dire de prix." (3)

Par conséquent, Diderot pense que l'aveugle enseigne le relativisme sur toutes ces questions dans lesquelles on adopte facilement un absolu.

D'autre part, Diderot répond à une question importante : Est-ce que la métaphysique de l'aveugle pourrait-elle ressemble à la nôtre? : "Combien de principes pour eux qui ne sont que des absurdités pour nous, et réciproquement!" (4) C'est ainsi que les merveilles de la nature n'ont guère de sens pour les aveugles ; et les grands raisonnements qu'on en tire leur doivent paraître bien faibles. Diderot dit à ce propos : "C'est ce grand raisonnement, qu'on tire des merveilles de la
(1) - Ibid.. pp.92.93
(2) - Ibid. p.95
(3) - Diderot(Denis): Lettre sur les aveugles ... ,op.cit. p.93
(4) - Idem.

(La Pensée matérialiste et la conception sensualiste) Dr. Siham Gabr 
nature, est bien faible pour des aveugles."(1) L'examen de l'aveugle permet de constater que les sens interviennent dans ce que l'on croit extra sensible ou super-sensible ou métaphysique. Il permet d'affirmer que la grande question, en ce qui concerne l'entendement, est de savoir comment l'homme passe des sensations aux signes communs entre tous, où commence mutuel des idées. Diderot dit : "Nos sens nous ramenant à des signes plus analogues à l'étendue de notre esprit et à la conformation de nos organes, Nous avons même fait en sorte que ces signes pussent être communs entre nous et qu'ils servissent? pour ainsi dire d'entre tôt au commence mutuel de nos idées." (2) En réalité, Diderot, essaye à travers La lettre sur les aveugles .., d'affirmer que la sensation n'est pas des sens eux-mêmes et qu'elle n'est pas non plus l'acte abstraitement appelé perception car elle est liée concrètement aux sens et aux objets.

Chez Diderot, la sensation penche vers l'objet en même temps que le mot se relie à un nom d'objet sensible. La sensation elle - même se localise dans cet objet, en rapport exprimé par différents termes, comme rapportés ou coïncidés : "La mémoire d'une suite de sensations du toucher, rapportées à la surface de quelque corps solide concave ...." (3) et Diderot ajoute : "... De n'apercevoir très distinctement que les limites de la sensation coüncidaient précisément avec celles de ces corps absents. " (4) L'aveugle
(1) - Ibid..p.93
(2) - Diderot(Denis): Lettre sur les aveugles ... _op.cit.p.93.
(3) -Ibid. p.95
(4) -Ibid. p.96

(La Pensée matérialiste et la conception sensualiste) Dr. Siham Gabr 
croit donc que la sensation n'est jamais autre que l'objet senti ; "L'aveugle-né ne discerne la sensation d'avec la présence réelle d'un objet à l'extrémité de son doigt que par la force ou la faiblesse de la sensation même." (1)

Cependant, Diderot montre aussi que cette collocation de la sensation reste à double sens : en même temps qu'elle se confond avec l'objet, elle est liée essentiellement à un organe corporel et que la sensation est la coexistence, non plus de l'âme et des objets, mais de l'organe, c'est-à-dire du corps et de la chose. C'est la forme qui articule les deux pôles de la réalité concrète : l'objet extérieur et l'ensablement d'un sens interne : l'auteur dit à ce propos : "C'est de là (du bout des doigts) que lui viennent ses principes sensations." (2)

Dans La lettre sur les aveugles..... la sensation est vue de côté de l'esprit, c'est que Diderot met le mot dans la bouche des idéalistes pour critiquer leur fausse appréciation de la dite sensation. Diderot a alors peint, dans sa lettre sur les aveugles, son système sensualiste par composition qui s'appuie principalement sur l'expérience et l'exercice. Il dit : "Plus généralement, il (l'aveugle) a par des expériences réitérées du toucher, la mémoire de sensations éprouvées en différents points : il est naître? de combiner ces sensations ou points et d'en former des figures." (3) Et Diderot ajoute :

\footnotetext{
(1) _Diderot(Denis): Lettre sur les aveugles ... _op.cit.. p.97

(2) - Idem.

(3) - Ibid. p.138
}

(La Pensée matérialiste et la conception sensualiste) Dr. Siham Gabr 
"C'est l'expérience seule qui nous apprend à comparer les sensations avec ce qui les occasionne." (1)

On peut dire ainsi que Diderot éclaire le problème de la mutation de la raison au XVIIIème siècle. La raison cède à l'instinct, à l'expérience, les sciences de la vie, ayant remplacé les sciences transcendantales. Pour Diderot, l'instinct est la source, le point de départ de l'expérience dans l'acte de l'observation des phénomènes de la nature. L'instinct se manifeste par l'appréhension directe de l'univers ou moyen des sens. Nous lisons dans La Lettre : "tous les animaux, nous accordant volontiers une raison avec laquelle nous aurions grand besoin de leur instinct, se prétendront doués d'un instinct avec lequel ils se passent fort bien de notre raison." (2) Diderot est, évidemment conscient des déformations de l'observation et de l'interprétation. Il dit : "Puisque les sens sont la source de toutes nos connaissances, il importerait beaucoup de savoir jusqu'où nous pouvons compter sur leur témoignage." (3)

En résumé, l'hypothèse et l'expérience pour Diderot sont inséparables. Il devance ainsi son temps, dans sa lettre,

\footnotetext{
(1) - Diderot(Denis): Lettre sur les aveugles ... _op.cit.p.138.

(2) -Ibid. p.89

(3) - Ibid..p.231
}

* Isaac Newton est un mathématicien, physicien, philosophe, alchimiste, astronome et théologien anglais, puis britannique. Wikipédia

* René Descartes est un mathématicien, physicien et philosophe français, né le 31 mars 1596 à La Haye-en-Touraine, baptisé le 3 avril 1596 dans l'église Saint-Georges de Descartes, et mort le 11 février 1650 à Stockholm. II est considéré comme l'un des fondateurs de la philosophie moderne. Wikipédia

(La Pensée matérialiste et la conception sensualiste) Dr. Siham Gabr 
en dépassant la méthode expérimentale de Newton* et la méthode rationnelle de Descartes*...

Diderot lance, en effet, une méthode qui enveloppe la raison et l'expérience et pose une problématique moderne de la connaissance où la méthodologie devient dialectique basée sur l'expérience et le raisonnement s'étayant sur l'autre au lieu de s'opposer.

Cependant, nous remarquons dans cette Lettre que Diderot ne conteste ni le pouvoir de l'imagination ni celui de la raison mais il exige que les deux soient soumis au contrôle de l'expérience. L'auteur voit que la pensée est soumise à un mouvement qui va du sujet à l'objet et de l'objet au sujet, du dedans au dehors, du subjectif à l'objectif, mouvement dialectique par excellence. L'acte perceptif est lié à l'acte intellectif, comme l'acte intellectif est lié à l'acte perceptif.

D'après Diderot, tout se réduit à revenir des sens à la réflexion aux sens.

C'est pourquoi il s'oppose au rationalisme de Descartes $^{(1)}$, au sensualisme de Condillac*(2) et d'Helvétius*(3) $^{*}$ à l'empirisme de Newton. Diderot explique

(1) _* René Descartes est un mathématicien, physicien et philosophe français, né le 31 mars 1596 à La Haye-en-Touraine, baptisé le 3 avril 1596 dans l'église Saint-Georges de Descartes, et mort le 11 février

(2) _* Étienne Bonnot de Condillac, abbé de Mureau, est un philosophe, écrivain, académicien et économiste français, né le 30 septembre 1714 à Grenoble et mort le 3 août 1780 à Lailly-en-Val. Wikipédia

(3) _* Claude-Adrien Helvétius ou Claude-Adrien Schweitzer, né le 26 janvier 1715 à Paris où il est mort le 26 décembre 1771, est un écrivain et philosophe français du courant des Lumières. Wikipédia

(La Pensée matérialiste et la conception sensualiste) Dr. Siham Gabr 
lui-même sa méthode comme suit : " un usage légitime de la raison et des sens appliquée à deux occupations bien pénibles:

-Recueillir les faits et lier les faits." ${ }^{(1)}$

Par conséquent, si l'activité de l'acte de connaissance demande à recueillir et à lier les faits en faisant un usage de la raison et des sens, cette activité passe, d'après lui, par trois étapes successives : "Il fallait appliquer les sens à la nature, l'entendement et l'expérience aux sens, et la troisième étape, non moins importante, appliquer l'entendement à l'entendement." (2)

D'ailleurs, Diderot trace une méthode générale par laquelle les aveugles peuvent s'instruire et se mettre en rapport : "Peut-être acquerraient des idées, si l'on se faisait entendre à eux dès l'enfance d'une manière fixe, déterminée, constante et uniforme, en un mot, si on leur traçait sur la main les mêmes caractères que nous traçons sur le papier et que la même signification leur demeurât invariablement attachée." (3)

Mais est-ce qu'on peut considérer cette méthode de Diderot comme l'une des théories du langage ? Il s'adresse dans sa Lettre à Mme Puiseaux son amie et lui dit : "Ce langage, Madame, ne vous parait-il pas aussi commode qu'un autre? N'est-il pas même tout inventé ? Il ne s'agit

(1) _Diderot(Denis): Lettre sur les aveugles ... . Textes établis par Paul Vernière.

Op.cit. p.191

(2) - Idem.

(3) -Diderot(Denis): Lettre sur les aveugles ... _op.cit.pp.99.100

(La Pensée matérialiste et la conception sensualiste) Dr. Siham Gabr 
donc que de le fixer et d'en faire une grammaire et des dictionnaires si l'on trouve que l'expression par les caractères ordinaires de l'écriture soit trop lente pour ce sens." ${ }^{(1)}$

En effet, Diderot fait allusion au triomphe du système sensualiste lorsqu'il dit dans sa Lettre : "J'ai lu avec toute l'attention dont je suis capable ce que Saunderson a dit de l'infini; je puis vous assurer qu'il avait su ce sujet des idées très justes et très nettes et que la plupart de nos infini aires n'auraient été pour lui que des aveugles." (2)

On remarque ainsi que le matérialisme de Diderot dans sa Lettre sur les aveugles a la conception sensualiste pour le dépasser, lui donner un sens nouveau et même la transformer en une théorie nouvelle de la connaissance.

Quant à la formation des figures, Diderot pense que pour l'aveugle, l'existence successive de sa main en plusieurs lieux, la sensation non interrompue d'un corps qui passe entre ses doigts, lui donne la notion de direction. S'il les glisse le long d'un fil bien tendu, il prend l'idée d'une ligne droite, s'il suit la courbure d'un fil lâche, il prend l'idée d'une ligne courbe. Par expérience du toucher, il a, en général, la mémoire des sensations énouées en différents points. C'est donc par la pratique continuelle qu'il peut combiner ses sensations et en former des figures contrairement au voyant qui peut former des idées et des figures par le concours de tous ses sens.
(1) - Ibid. p.100
(2) - Ibid.p. 115

(La Pensée matérialiste et la conception sensualiste) Dr. Siham Gabr 
D'autre part, Diderot montre que les aveugles ne sont pas complètement infirmes car leurs longues expériences sensualistes et pratiques continuelles, peuvent les rendre capables de faire des travaux difficiles comme l'utilisation de l'aiguille, l'exécution d'un morceau musical et la lecture par des caractères en relief.

Cette conception sensualiste de Diderot dans la Lettre que nous étudions résume ce qu'on peut facilement appeler sa théorie de connaissance en trois points :-

I- La connaissance d'après lui, résulte de l'activité pratique et non pas d'une somme de sensations passives et individuelles.

II- La connaissance est un fait social et historique .

III- Le rationalisme métaphysique a tort, car la connaissance vient des choses et en même temps de notre activité puisque c'est cette activité qui découvre les choses en les pénétrant et les organisant.

Pour conclure, on peut dire que ce grand écrivain du dixhuitième siècle et ce grand penseur a lancé au nom de Nicholas Saunderson, célèbre mathématicien anglais, aveugle de naissance qui a vécu de 1682 à 1739 et qui a imaginé à l'âge de 25 ans une arithmétique palpable, une nouvelle conception de la vie. L'aveugle choisi par Diderot pour être le sujet de la Lettre sur les aveugles à l'usage de ceux qui voient n'est pas un aveugle normal mais c'est un aveugle raisonneur. Il n'est pas ingénu comme la plupart des aveugles ordinaires. D'où son importance dans le 
développement des idées philosophiques de Diderot expliquées par les différents personnages de la Lettre.

\section{Conclusion:}

Nous avons essayé en analysant les deux tendances : matérialiste et sensualiste dans cet ouvrage de Diderot : Lettre sur les aveugles à l'usage de ceux qui voient, ouvrage presque inaperçu, de mettre en exergue deux tendances ou deux aspects répandus au siècle des lumières. Nous avons certainement remarqué comment Diderot adoptait la forme d'un récit imaginaire pour prêter au mathématicien anglais Saunderson, qui avait perdu la vue, ses propres arguments, sa faveur de l'athéisme .

Diderot, comme nous l'avons remarqué, conteste dans sa Lettre sur les aveugles à l'usage de ceux qui voient, les preuves traditionnelles de l'existence de Dieu ; car que valent pour un aveugle l'ordre du monde et les merveilles de la nature. Il dit : "Laissez là tout ce beau spectacle qui n'a jamais été fait pour nous. J'ai été condamné à passer ma vie dans les ténèbres ; et vous me citez des prodiges que je n'entends point et qui ne prouvent que pour vous et que pour ceux qui voient comme vous." (1)

Diderot pense, d'ailleurs, que pour croire en l'existence de Dieu, il faut que l'on puisse toucher.

Nous avons certainement vu que Diderot, en exposant ses idées matérialistes et sensualistes, s'appuie sur une dialectique philosophique et scientifique.

(1) _Diderot(Denis): Lettre sur les aveugles ... Op.cit. p.100

(La Pensée matérialiste et la conception sensualiste) Dr. Siham Gabr 
Nous voudrions signaler que d'autres ont abordé les sujets traités dans cette Lettre sur les aveugles comme Condillac dans son ouvrage, Essai sur l'origine des connaissances $^{(1)}$ et dans son traité des sensations ${ }^{(2)}$

Nous trouvons aussi Julien Offray de la Mattris qui avait écrit en 1748 un livre intitulé L'homme-Machine où les lecteurs découvrent une certaine ressemblance avec la Lettre sur les aveugles de Diderot ; car les deux textes manifestent le même mouvement d'écriture puisque chacun croit que la pensée dépend étroitement de la matière.

Disons brièvement que le caractère cohérant de son matérialisme, dû au sensualisme, est presque identique à la conception de Diderot.

Nous remarquons aussi une conception presque identique à celle de Diderot dans sa Lettre sur les aveugles, dans l'œuvre du penseur Helvétius intitulée : De l'esprit.

Malgré tout, nous pouvons dire que Diderot ne compte pas de philosophies le matérialisme ou le sensualisme quoiqu'il lance une théorie ou une conception basée sur le matérialisme et le sensualisme, plus fondée et plus sure que les idées des penseurs déjà cités.

\footnotetext{
(1) _Condillac (Etienne ) :Essai sur l'origine des connaissances humaines. La Pleiade 1920 p .1746

(2)_Condillac (Etienne ) : Traité des sensations. . La Pléiade 1920 pp .1709-1751
}

(La Pensée matérialiste et la conception sensualiste) Dr. Siham Gabr 


\section{Bibliographie}

\section{I-Corpus:}

-Diderot(Denis):- Lettre sur les aveugles à l'usage de ceux qui voient . Paris. La pléiade .1935

-Lettre sur les aveugles à l'usage de ceux qui voient:Texte établis par Vernière (p.).Paris.Garnier 1963

-Euvres Complètes.Paris Le Club Français du livre.1973

II-Ouvrages généraux

1-Candillac(Etienne):Traité des sensations .Paris .Collection des chefs d'œuvre méconnus.1920.

2-Delon( Michel):Diderot et ses artistes . Paris .Gallimard. 2013

3-Descartes ( R. ) : Discours de la méthode . Paris . Garnier . 1960.

4- Diderot ( D. ): De l'interprétation de la nature . Paris Garnier . 1964.

5- Diderot ( D. ): Entretiens entre D'Alembert et Diderot. Paris. Garnier 1966.

6-Ducros (louis): " Diderot, l'homme et l'écrivain" Paris, Perrier ,1894.

7-Helvétius(Claude Adrien) : De l'esprit, Paris,Garnier,1758.

8- Le Breton (André): de roman au XVIII ${ }^{\mathrm{e}}$ siècle, Paris, Société françaises d'imprimerie et de libraire, 1898.

9- Mornet (Daniel): " La pensée Francaise au XVIII ${ }^{\mathrm{e}}$ Siècle," Paris, A. Colin, 1969 -.

(La Pensée matérialiste et la conception sensualiste) Dr. Siham Gabr 
10-Voltaire(François-Arouet) : Elements de la philosophie de Newton. Paris . Garnier.1738.

\section{III-Ouvrages sur Diderot (D.) et La lettre sur les aveugles à l'usage de ceux qui voient.}

1- Callot(Emile): six philosophes français du XVIIIème siècle .L'œuvre et la doctrine de Diderot .Annecy Gardet.1962. Voir les pages 7-38

2- Cresson (A.): Diderot, sa vie, ses œuvres et sa philosophie. Paris. P.U.F, 1949.

3- Ehrard ( Jean): Matérialisme et naturalisme : les sources occultistes de la pensée de Diderot .Paris .Cahiers de l' A.I.E.F No 13 .1966 .pp.189-201.

4- Fontenoy (Elisabeth) : Diderot, ou le matérialisme enchanté. Paris .Grasset.1981.

5- Lefebvre (Henri) : Le matérialisme dialectique. Paris, P.U.F 1949.

6- Naigeon (Jacques) :Mémoires historiques et philosophiques sur la vie et les ouvrages de Denis Diderot .Genève . Slatkine. 1970.

7- Potulioki(Elisabeth): La modernité de la pensée de Diderot dans les œuvres philosophiques .Vizet 1980.

8- Rostand (Jean) : La conception de l'homme selon Helvétuis et selon Diderot .Paris in .Revue d'histoire des Sciences et de leurs applications, tome 4, n³-4, 1951. pp. 213-222

9- Szigeti (Jozef) : Denis Diderot, une grande figure du matérialisme militant du XVIIIème siècle. Budabest,Academie Kiado .1977.

(La Pensée matérialiste et la conception sensualiste) Dr. Siham Gabr 


\section{Sitographie}

-fr.wikisource.org > wiki > Euvres_complètes_de_Diderot -https://www.espacefrancais.com/les-oeuvres-de-denisdiderot/ -fr.wikipedia.org > wiki > Denis_Didero -fr.wikipedia.org > wiki > Lettre_sur_les_aveugles_à_l'u... -homepages.uc.edu $>\sim$ martinj > French > Diderot - Lettr... editions.flammarion.com $>$ Catalogue $>$ philosophie > -www.cairn.info > revue-le-philosophoire-2003-3-page-... -livre.fnac.com > Denis-Diderot-Lettre-sur-les-aveugles-... -psychaanalyse.com > pdf > DIDEROT BIBLIO LETTR... -homepages.uc.edu $>\sim$ martinj > French > Diderot - Lettr... -d1n7iqsz6ob2ad.cloudfront.net $>$ document $>$ pdf -gallica.bnf.fr > essentiels > diderot > lettre-sur-les-aveug... -fr.wikisource.org > wiki > Notice -www.researchgate.net > publication > 317401917_Ecrire_... 


\section{الفكر المادي والمفهوم الحسي للكاتب ديلرو في روايه" رساله حول

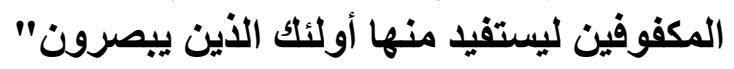

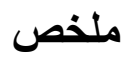

تلقى ديدرو تعليمًا قويًا من سن 1 ( ووقد كانت هذه التعليمات أساس ثقافته المذهلة. في رسالته حول المكفوفين ليستفيد منها أولئك الذين ييصرون ، يحاول ديدرو إطلاق مفهوم لعالمنا ، أو طريقة جديدة ، أو من الأفضل القول ، نظرية شاملة ؛

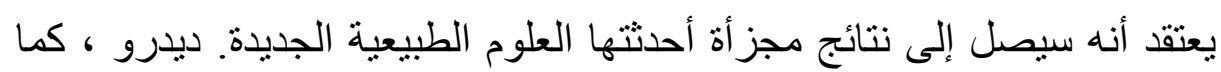
لاحظنا ، يجادل في عمله من اجل اثبات البراهين التقليدية لوجود اله ؛ فما هو

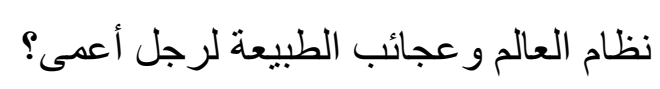

على الرغم من كل شيء ، يمكننا القول أن ديدرو لا يحسب المادية أو فلسفات الحسية على الرغم من أنه أطلق نظرية أو مفهومًا قائمًا على المادية والحسية ؛ أكثر ترسخًا وأكثر يقينًا من أفكار المفكرين التي تم الاستشهاد بها بالفعل . هذا ، في الواقع ، و غني عن البيان أن أفكار ديدرو الواردة في هذه الرسالة قد تم الطعن فيها ومناقشتها من قبل العديد من المفكرين الآخرين. ستتطلب مثل هذه

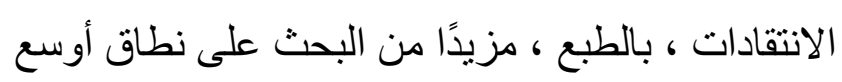

